\title{
DELEGATED DYNAMIC PORTFOLIO MANAGEMENT UNDER MEAN-VARIANCE PREFERENCES
}

\author{
COSKUN CETIN
}

Received 24 January 2006; Revised 12 March 2006; Accepted 7 June 2006

We consider a complete financial market with deterministic parameters where an investor and a fund manager have mean-variance preferences. The investor is allowed to borrow with risk-free rate and dynamically allocate his wealth in the fund provided his holdings stay nonnegative. The manager gets proportional fees instantaneously for her management services. We show that the manager can eliminate all her risk, at least in the constant coefficients case. Her own portfolio is a proportion of the amount the investor holds in the fund. The equilibrium optimal strategies are independent of the fee rate although the portfolio of each agent depends on it. An optimal fund weight is obtained by the numerical solution of a nonlinear equation and is not unique in general. In one-dimensional case, the investor's risk is inversely proportional to the weight of the risky asset in the fund. We also generalize the problem to the case of multiple managers and provide some examples.

Copyright (C 2006 Coskun Cetin. This is an open access article distributed under the Creative Commons Attribution License, which permits unrestricted use, distribution, and reproduction in any medium, provided the original work is properly cited.

\section{Introduction and problem formulation}

In this paper, we consider a delegated portfolio management problem in a complete financial market where a small investor does not have direct access to the risky assets in an equity market (due to high transaction costs, taxes, or other constraints). So the investor trades dynamically through a mutual fund by paying an instantaneous fee to a fund manager. For a recent (and the first to our knowledge) work on the relationship between fund flows and fund returns in a dynamic setting, one may see Hugonnier and Kaniel [6] where the investor has a log utility and the manager has an increasing and strictly concave utility. Our setup differs from theirs mainly in the choice of utility functions for the agents, the extension to the multiple fund managers case, and the explicit characterization of manager's strategy. We assume that both the investor and manager have mean-variance preferences which result in different optimal allocations even in the 
deterministic coefficients case. The fund portfolio weights depend on the preferences of the manager and influence the value function of the investor. Therefore, investor would benefit from a competition among multiple mutual funds. Because of the short selling constraints, investor faces an incomplete market situation, and the quadratic optimization techniques using martingale duality or Riccati BSDEs are not easily applicable to our case. One may refer to Lim [10] for recent applications and shortcomings of Riccati BSDEs.

In this work, we mainly consider the deterministic coefficients case where dynamic programming principle (DPP) would apply to the problem of the investor. We show that given the fund portfolio proportions by the manager, the optimal portfolio of the investor is a feedback control depending on the wealth of the investor and the fund's net of the fees Sharpe ratio. The optimal wealth process is a viscosity solution to the corresponding Hamilton-Jacobi-Bellman (HJB) equation. We characterize the manager's optimal strategies (both her own portfolio and the fund's portfolio) through martingale duality method thanks to the market completeness. We also provide some numerical examples with financial interpretation and generalize the results to the case of multiple fund managers.

Throughout the paper, we only consider the case of the fixed fee rate and do not discuss the optimal compensation contracts and performance fees. We refer the reader to OuYang [13], Cvitanić et al. [4] and references therein for a discussion of such concepts in the setting of principal-agent problems. In the multiple managers case, we assume that the investor chooses only one of the managers to minimize his risk. In a more general and realistic setup, the investor would consider a combination of various managers. However, this generalization is not discussed here, either.

1.1. The model. Let $\left\{\digamma_{t}, 0 \leq t \leq T\right\}$ be the complete $\sigma$-algebra generated by an $n$-dimensional Brownian motion $B=\left(B_{1}, \ldots, B_{n}\right)$ on a probability space $(\Omega, \digamma, P)$. Assume that $r$, $\mu$, and, $\sigma$ are $\mathbb{R}, \mathbb{R}^{n}$, and $\mathbb{R}^{n \times n}$ valued continuous parameters on $[0, T]$, respectively, such that $\sigma^{-1}$ and $\theta=\sigma^{-1}(\mu-r 1)$ are uniformly bounded and $\mu_{i}-r>0,1 \leq i \leq n$, on $[0, T]$. We introduce the "state price density" process $H$ as $H(t) \triangleq \exp \left(-\int_{0}^{t} r(s) d s\right) Z_{0}(t), 0 \leq t \leq$ $T$, where $Z_{0}(t)=\exp \left(-\int_{0}^{t} \theta(s)^{\prime} d B(s)-(1 / 2) \int_{0}^{t}|\theta(s)|^{2} d s\right)$ is a strictly positive martingale and the prime $\left({ }^{\prime}\right)$ denotes the transpose of a matrix. The risk neutral probability measure $P^{0}$ is given by $P^{0}(A)=E\left[1_{A} Z_{0}(T)\right]$ under which the process $B^{0}(t) \triangleq B(t)+\int_{0}^{t} \theta(s) d s$ is an $n$-dimensional Brownian motion. The risk-free asset $S_{0}$ and the $n$ risky assets $S_{i}, 1 \leq i \leq n$, satisfy

$$
\begin{gathered}
d S_{0}(t)=r(t) S_{0}(t) d t, \quad S_{0}(0)=1 \\
d S_{i}(t)=S_{i}(t)\left(\mu_{i}(t) d t+\sum_{j=1}^{n} \sigma_{i j}(t) d B_{j}(t)\right), \quad S_{i}(0)=s_{i}, 0 \leq t \leq T .
\end{gathered}
$$

Then the discounted process $\bar{S}(\cdot) \triangleq S(\cdot) / S_{0}(\cdot)$ with $S(t)=\left(S_{1}(t), \ldots, S_{n}(t)\right)^{\prime}$ is a martingale with respect to $P^{0}: d \bar{S}_{i}(t)=\bar{S}_{i}(t) \sum_{j=1}^{n} \sigma_{i j}(t) d B_{j}^{0}(t), i=1, \ldots, n, 0 \leq t \leq T$. We also 
define $L_{\digamma}^{p}\left([0, T], \mathbb{R}^{n}\right)$ (resp., $\left.L_{\digamma}^{\infty}\left([0, T], \mathbb{R}^{n}\right)\right)$ to be the set of all $\digamma$-adapted $\mathbb{R}^{n}$ valued processes $u$ such that $E\left[\int_{0}^{T}|u(t)|^{p} d t\right]<\infty$ (resp., $u$ is essentially bounded on $\left.[0, T]\right)$. When it is clear from the context, we write $L_{\digamma}^{p}\left(\mathbb{R}^{n}\right)$ (resp., $\left.L_{\digamma}^{\infty}\left(\mathbb{R}^{n}\right)\right)$.

1.2. Dynamics for the fund, investor, and manager. We first study the case of a single fund manager who decides both the fee rate and the fund portfolio. Given an $n$ dimensional portfolio weight process (dollar proportions) $\pi(t)$ by the manager, the value of the fund for one dollar invested satisfies

$$
d F(t)=F(t)\left[r(t)+\pi^{\prime}(t) \sigma(t) \theta(t)\right] d t+F(t) \pi^{\prime}(t) \sigma(t) d B(t), \quad 0 \leq t \leq T .
$$

Our emphasis in this paper is mainly on the portfolio weight processes for which both the fund value and the net of the fees Sharpe ratio $\left(\pi^{\prime} \sigma \theta-\gamma\right) /\left|\sigma^{\prime} \pi\right|^{2}(t)$ of the fund are nonnegative, where $\gamma \in(0,1)$ is the fee rate determined in advance. We will discuss the significance of such portfolio weights in the next section.

The cumulative discounted fee process $\Phi(t)$ that represents the market value of the manager's compensation for management services is given by $\Phi(t)=\int_{0}^{t} \gamma \exp \left(-\int_{0}^{s} r(u) d u\right)$ $\phi(s) d s$, where $\phi(s)$ is the dollar amount that the investor keeps in the fund at time $t=s$. The investor is allowed to change the amount $\phi$ in the fund dynamically provided $\phi$ stays nonnegative. Given an admissible $\pi$ by the manager and the investor's corresponding portfolio $\phi^{\pi} \geq 0$, the dynamics of the investor's wealth process can be written as

$$
\begin{aligned}
d W(t) & =\left(W r+\phi \pi^{\prime} \sigma \theta\right)(t) d t+\phi \pi^{\prime} \sigma(t) d B(t)-\gamma \phi(t) d t, \\
& =(W r-\gamma \phi)(t) d t+\phi \pi^{\prime} \sigma(t) d B^{0}(t), \quad 0 \leq t \leq T .
\end{aligned}
$$

Remark 1.1. The admissibility criterion can be restricted to the portfolios $\phi$ for which the wealth $W(t)$ is bounded from below: $W(\cdot) \geq M$, for some $M$. For a recent work on a portfolio selection problem under bankruptcy constraint, see [1].

We assume that the investor has mean-variance preferences over the terminal wealth. His objective is to minimize the variance $E\left[(W(T)-c)^{2}\right]$ subject to $E[W(T)]=c$ and $c>w \exp \left(\int_{0}^{T} r(s) d s\right)$. If $c \leq w \exp \left(\int_{0}^{T} r(s) d s\right)$, he would just invest in the risk-free bond. By introducing a Lagrange multiplier $\lambda \geq 0$, this problem can be written in the form

$$
\begin{aligned}
\Gamma(\lambda ; \pi) & \triangleq \inf _{\phi \geq 0}\left\{E\left[(W(T)-c)^{2}\right]-2 \lambda(E[W(T)]-c)\right\} \\
& =\inf _{\phi \geq 0} E\left[(W(T)-(c+\lambda))^{2}\right]-\lambda^{2}
\end{aligned}
$$

that is similar to a portfolio selection problem studied by Li et al. [9].

As being the strategic leader in this setup, the manager decides the fund's strategy $\pi$ as well as her own portfolio $\psi_{M}$. She receives the cumulative fee process $\int_{0}^{t} \gamma \phi(s) d s$ from 
the investor, and her wealth process $W_{M}$ with $W_{M}(0)=w_{M}$ satisfies the dynamic budget constraint

$$
\begin{aligned}
d W_{M}(t) & =\left(W_{M} r+\psi_{M}^{\prime} \sigma \theta\right) d t+\psi_{M}^{\prime} \sigma d B(t)+\gamma \phi d t, \\
& =\left(W_{M} r+\gamma \phi\right) d t+\psi_{M}^{\prime} \sigma d B^{0}(t), \quad 0 \leq t \leq T .
\end{aligned}
$$

She has mean-variance preferences and her aim is to minimize $E\left[\left(W_{M}(T)-c_{M}\right)^{2}\right]$ subject to the constraint $E\left[W_{M}(T)\right]=c_{M}$ with $c_{M}>w_{M} \exp \left(-\int_{0}^{T} r(s) d s\right)>0$.

\section{Optimal trading strategies}

We assume that the agents observe the actions of each other and the fee rate $\gamma$ is constant. As in [6], the portfolio allocation problem with a single manager can also be viewed as a stochastic game where the manager is a Stackelberg leader and the equilibrium is achieved as follows. First, the investor solves his optimization problem given $\pi$ by the manager. Then, the manager solves her own optimization problem. Finally, she solves for the optimal fund strategy from her perspective.

Notation 2.1. Let $\Pi$ denote the set of the bounded deterministic portfolio weights $\pi$ such that $\left|\sigma^{\prime} \pi\right|^{-1}(\cdot)$ is bounded on $[0, T]$ and that the $\operatorname{SDE}(1.2)$ has a unique solution. Set

$$
\rho(t) \triangleq \frac{\pi^{\prime} \sigma \theta-\gamma}{\left|\sigma^{\prime} \pi\right|^{2}}(t), \quad \xi(t) \triangleq\left(\frac{\pi^{\prime} \sigma \theta-\gamma}{\left|\sigma^{\prime} \pi\right|}\right)^{2}(t)
$$

for $0 \leq t \leq T$, where $\rho(t)$ represents after the fees Sharpe ratio of the fund.

2.1. The investor's problem. We introduce a new process $X$ as follows:

$$
X(t ; \lambda) \triangleq W(t)-(c+\lambda) \exp \left(-\int_{t}^{T} r(s) d s\right), \quad 0 \leq t \leq T .
$$

Then the dynamics of $X$ is

$$
\begin{gathered}
d X(t)=\left(X r+\phi\left(\pi^{\prime} \sigma \theta-\gamma\right)\right) d t+\phi \pi^{\prime} \sigma d B(t), \quad 0 \leq t \leq T \\
X(0)=x_{0}=w-(\lambda+c) \exp \left(-\int_{0}^{T} r(s) d s\right)<0,
\end{gathered}
$$

and the optimization problem (1.4) can be rephrased as follows: consider the cost function $J^{\pi}(s, x ; \phi)=E\left[X^{\pi, \phi}(T)^{2}\right]$ with $X(s)=x$, and define

$$
\begin{aligned}
V^{\pi}(s, x) & =\inf _{\phi \geq 0} J^{\pi}(s, x ; \phi), \\
\Gamma(\lambda ; \pi) & =V^{\pi}\left(0, x_{0}\right)-\lambda^{2},
\end{aligned}
$$

where the initial condition $x_{0}=x_{0}(\lambda)$ in (2.4) depends on $\lambda$. In the rest of the paper, we will usually suppress the dependence on $\lambda, \pi$, and $\phi$, for the simplicity of the presentation. 
Now, fixing $\lambda \geq 0$, the value function $V(t, x)$ with the terminal condition $V(T, x)=x^{2}$ satisfies the HJB equation

$$
0=V_{t}+r x V_{x}+\inf _{\phi \geq 0}\left\{\phi\left(\pi^{\prime} \sigma \theta-\gamma\right) V_{x}+\frac{1}{2} \phi^{2}\left|\sigma^{\prime} \pi\right|^{2} V_{x x}\right\}
$$

This PDE does not necessarily have a smooth solution due to the short selling constraint $\phi \geq 0$. However, we will show below that the viscosity solution to the PDE (2.7) can be obtained when $\pi$ is restricted to some deterministic portfolio weights.

Definition 2.2. Let $\hat{\phi}^{\pi} \in L_{\digamma}^{2}(\mathbb{R})$ be an optimal portfolio of the investor for $\pi \in \Pi$. Then the set of the feasible fund portfolio weights is defined by $\Pi_{f}=\left\{\pi \in \Pi: E\left[W^{\pi, \hat{\phi}^{\pi}}(T)\right] \geq c\right\}$.

We first characterize the optimal strategy of the investor when $\pi \in \Pi_{f}$ via a viscosity solution to the HJB equation (2.7). Then, we consider the random parameters case when $\pi$ is a bounded adapted process satisfying $\rho^{\pi}(t)>0$.

Proposition 2.3. Let $\pi \in \Pi_{f}$ and $\lambda \geq 0$ be given, and let $\rho$ and $\xi$ be as in (2.1). Define

$$
\begin{gathered}
A(t)=\exp \left(\int_{t}^{T} 2 r(s) d s\right), \quad a(t)=A(t) \exp \left(\int_{t}^{T}(-\xi)(s) d s\right), \\
\phi(t, x)=\left(-\rho(t) \frac{v_{x}}{v_{x x}}\right)^{+}=(-\rho(t) x)^{+}, \quad 0 \leq t \leq T .
\end{gathered}
$$

Then the HJB PDE (2.7) has a viscosity solution $v(t, x)$ which is given by

$$
v(t, x)= \begin{cases}a(t) x^{2}, & x \rho(t) \leq 0, \\ A(t) x^{2}, & x \rho(t)>0,\end{cases}
$$

and the control $\{\phi(t, X(t)): X(s)=x, t \geq s\}$ is optimal for the problem (2.5).

Proof. See Appendix A.

Remark 2.4. Note that the optimal state process with $X(0)=x_{0}<0$ and dynamics

$$
d X(t)= \begin{cases}X(r-\xi) d t-X \rho \pi^{\prime} \sigma d B(t), & \rho(t)>0, \\ X r d t, & \rho(t) \leq 0\end{cases}
$$

has the unique solution $X(t)=x_{0} \exp \left(\int_{0}^{t}(r-(3 / 2) \xi)(u) d u-\int_{0}^{t} \rho \pi^{\prime} \sigma d B(u)\right)$ which is negative a.s. on $[0, T]$ when $\pi \in \Pi_{f}$. Therefore, Proposition 2.3 simplifies to the following.

Corollary 2.5. Let $\pi \in \Pi_{f}$ be given. Then the value function $V^{\pi}(t, x)$ satisfies

$$
V(t, x)= \begin{cases}a(t) x^{2}, & \rho(t)>0, \\ A(t) x^{2}, & \rho(t) \leq 0,\end{cases}
$$


with the optimal control

$$
\widehat{\phi}(t, X(t))=(-\rho(t) X(t))^{+}
$$

where $a(\cdot)$ and $A(\cdot)$ are given by (2.8). If $\rho(\cdot)>0$ on $[0, T]$, then $V(t, x)=a(t) x^{2}$ is $a$ smooth solution of the PDE (2.7) with the optimal control $\hat{\phi}(t, X(t))=-\rho(t) X(t)$.

Remark 2.6. Since $a \leq A$ on $[0, T]$, the investor prefers the fund portfolio weights with $\rho(\cdot)>0$ to minimize $V(t, x)$ in (2.12). When $\rho(t) \leq 0$, he does not invest in the fund by (2.13) and the manager does not get any compensation. So, it is not an optimal strategy for the manager to choose such fund portfolio weights $\pi$ which are not feasible in the sense of Definition 2.2, either. Hence, the condition $\rho(\cdot)>0$ is a reasonable assumption from the perspectives of both agents. These portfolio weights also allow us to extend the results above to the random parameters case using martingale duality method.

Definition 2.7. The set $\mathcal{U}$ of admissible control processes is defined by

$u \triangleq\left\{\phi \in L_{\digamma}^{2}(\mathbb{R}): \phi \geq 0, X \leq 0\right.$, a.s. on $[0, T]$ and the $\operatorname{SDE}(2.3)$ has a unique solution $\}$.

We then have the following result.

Proposition 2.8. Assume that all the model parameters $\mu, r, \sigma$, and $\theta$ are $\digamma_{t}$-adapted and uniformly bounded on $[0, T]$. Let $\lambda \geq 0$ and $\pi \in \Pi_{f}$ with $\rho(\cdot)>0$ a.s. on $[0, T]$. Then the optimization problem (2.5) has the solution

$$
V(s, x)=x^{2} \exp \left(\int_{s}^{T}(2 r-\xi)(u) d u\right)
$$

with an optimal control

$$
\widehat{\phi}(t)=-\rho(t) X(t)>0, \quad \text { a.s. }
$$

where $X$ satisfies (2.3)-(2.4) and $\hat{\phi} \in \mathcal{U}$.

Proof. See Appendix B.

Remark 2.9. The martingale duality is a standard tool to characterize the optimal terminal condition in a complete market setting. The condition $\rho(\cdot)>0$ reduces the constrained optimization problem (2.5) to an unconstrained one (as in the proof of Proposition 2.8). So the duality argument works as if the investor faces a complete market. For more information on the theory and applications of martingale duality method, one can refer to $[3,7]$.

Now, we turn back to the original problem: for any given $\lambda \in \mathbb{R}$ and $\pi \in \Pi_{f}$, let $\Gamma(\lambda ; \pi)$ be the optimization problem given by (2.6). Then by (2.12), we get

$$
\Gamma(\lambda ; \pi)=V\left(0, x_{0}(\lambda)\right)-\lambda^{2}=a(0) x_{0}^{2}-\lambda^{2} .
$$


After substituting $a(0)$ into the formula and using $0>x_{0}=w-(c+\lambda) \exp \left(-\int_{0}^{T} r(s) d s\right)$ with $\xi(t)=\left(\left(\pi^{\prime} \sigma \theta-\gamma\right)^{2} /\left|\sigma^{\prime} \pi\right|^{2}\right)(t),(2.17)$ results in a concave quadratic function:

$$
\begin{aligned}
V\left(0, x_{0}\right)-\lambda^{2} & =a(0)\left[w-(c+\lambda) \exp \left(-\int_{0}^{T} r(s) d s\right)\right]^{2}-\lambda^{2} \\
& =\exp \left(-\int_{0}^{T} \xi(s) d s\right)\left[w \exp \left(\int_{0}^{T} r(s) d s\right)-(c+\lambda)\right]^{2}-\lambda^{2}
\end{aligned}
$$

Now, we can find the optimal $\lambda$ by solving the equation

$$
c=E[W(T ; \lambda)]=E[X(T ; \lambda)]+(c+\lambda)
$$

for $\lambda$, where

$$
\begin{aligned}
E[X(T ; \lambda)] & =x E\left[\exp \left(\int_{0}^{T}\left(r-\frac{3}{2} \xi\right)(s) d s-\int_{0}^{T} \rho \pi^{\prime} \sigma(s) d B_{s}\right)\right] \\
& =\left[w-(c+\lambda) \exp \left(-\int_{0}^{T} r(s) d s\right)\right] \exp \left(\int_{0}^{T}(r-\xi)(s) d s\right) .
\end{aligned}
$$

By (2.18), (2.19), and (2.20), the optimal values are

$$
\left(\lambda^{*}, \Gamma\left(\lambda^{*} ; \pi\right)\right)=\left(\frac{c-w \exp \left(\int_{0}^{T} r(s) d s\right)}{\exp \left(\int_{0}^{T} \xi(s) d s\right)-1}, \frac{\left(c-w \exp \left(\int_{0}^{T} r(s) d s\right)\right)^{2}}{\exp \left(\int_{0}^{T} \xi(s) d s\right)-1}\right) \text {. }
$$

Remark 2.10. By following similar arguments above, or using martingale duality method as in [2], one can show that when investor has no constraints (has direct access to the market), the corresponding optimal values are

$$
(\tilde{\lambda}, \Gamma(\tilde{\lambda}))=\left(\frac{c-w \exp \left(\int_{0}^{T} r(s) d s\right)}{\exp \left(\int_{0}^{T}|\theta(s)|^{2} d s\right)-1}, \frac{\left(c-w \exp \left(\int_{0}^{T} r(s) d s\right)\right)^{2}}{\exp \left(\int_{0}^{T}|\theta(s)|^{2} d s\right)-1}\right),
$$

where $\Gamma(\tilde{\lambda})<\Gamma\left(\lambda^{*} ; \pi\right)$ since $\int_{0}^{T}|\theta(s)|^{2} d s>\int_{0}^{T} \xi(s)^{2} d s$, for any $\gamma>0$ and $\pi \in \Pi_{f}$.

Hence, the optimal portfolio $\hat{\phi}(t)=-\rho(t) X(t)$ of the investor can be written as

$$
\hat{\phi}(t)=\rho(t)\left[\left(c+\lambda^{*}\right) \exp \left(-\int_{t}^{T} r(s) d s\right)-W(t)\right]>0, \quad \text { a.s. },
$$

with

$$
c+\lambda^{*}=\frac{c \exp \left(\int_{0}^{T} \xi(s) d s\right)-w \exp \left(\int_{0}^{T} r(s) d s\right)}{\exp \left(\int_{0}^{T} \xi(s) d s\right)-1}
$$


Corollary 2.11. For $\pi \in \Pi_{f}$ and $c>w \exp \left(\int_{0}^{T} r(s) d s\right)$, the efficient frontier for the investor's terminal wealth is given by

$$
(E[W(T)], \operatorname{Var}(W(T)))=\left(c, \frac{\left(c-w \exp \left(\int_{0}^{T} r(s) d s\right)\right)^{2}}{\exp \left(\int_{0}^{T} \xi(s) d s\right)-1}\right) .
$$

Remark 2.12. For one-dimensional constant parameters case, the functional $\Gamma\left(\lambda^{*} ; \pi\right)=$ $\left(c-w \exp \left(\int_{0}^{T} r(s) d s\right)\right)^{2} /\left(\exp \left(\int_{0}^{T} \xi(s) d s\right)-1\right)$ becomes $\left(c-w e^{r T}\right)^{2} /(\exp (\xi T)-1)$, that is, a convex decreasing (concave increasing, resp.) function of $\pi \in(\gamma /(\mu-r), \infty)\left(c \in\left(w e^{r T}\right.\right.$, $\infty)$, resp.).

2.2. Manager's problem. Given the best response $\phi^{\pi}(t)$ of the investor to the portfolio weight process $\pi$, the manager's own portfolio $\psi^{\pi}(t)$ can be optimized by using the standard duality methods taking the advantage of the market completeness. Some of the following arguments and notation are also similar to the ones in [6].

By Corollary 2.5, the cumulative fee process is

$$
\Phi^{\pi}(t)=\int_{0}^{t} \gamma \phi^{\pi}(u) \exp \left(-\int_{0}^{u} r(s) d s\right) d u=-\int_{0}^{t} \gamma \rho(u) X^{\pi}(u) d u
$$

for $\pi \in \Pi$ and $X^{\pi} \in L_{\digamma}^{2}\left(\mathbb{R}^{n}\right)$. Now, setting

$$
v^{\pi}(t) \triangleq E_{t}^{0}\left[\int_{t}^{T} d \Phi^{\pi}(u)\right]=\gamma E_{t}^{0}\left[\int_{t}^{T} \exp \left(-\int_{t}^{u} r(s) d s\right) \phi^{\pi}(u) d u\right],
$$

we get $v^{\pi}(t)=\gamma \int_{t}^{T} \exp \left(-\int_{t}^{u} r(s) d s\right) \rho(u) E_{t}^{0}[X(u)] d u$.

Definition 2.13. Let $\psi \in L_{\digamma}^{2}\left(\mathbb{R}^{n}\right), \pi \in \Pi_{f}$, and let $W_{M}=W_{M}^{\psi}$ be as in (1.5). Then, $\left(\psi, W_{M}^{\psi}(T)\right)$ satisfy the static budget constraint for the manager if the condition

$$
E^{0}\left[\exp \left(-\int_{0}^{T} r(s) d s\right) W_{M}(T)\right] \leq v^{\pi}(0)+w_{M}
$$

holds. Also define the set of the admissible portfolio processes for the manager, denoted by $\Psi^{\pi}$, as follows:

$$
\Psi^{\pi}=\left\{\psi \in L_{\digamma}^{2}\left(\mathbb{R}^{n}\right): \text { the } \operatorname{SDE}(1.5) \text { has a unique solution and (2.28) is satisfied }\right\} .
$$

For a given $\pi \in \Pi_{f}$, and $\lambda_{M} \geq 0$, we then define

$$
\begin{aligned}
V_{M}^{\pi}\left(\lambda_{M}\right) & =\inf _{\psi \in \Psi^{\pi}} E\left[\left(W_{M}(T)-c_{M}\right)^{2}\right]-2 \lambda_{M}\left(E\left[W_{M}(T)\right]-c_{M}\right) \\
& =\inf _{\psi \in \Psi^{\pi}} E\left[\left(W_{M}(T)-\left(c_{M}+\lambda_{M}\right)\right)^{2}\right]-\lambda_{M}^{2} .
\end{aligned}
$$

Since $\lambda_{M}$ is independent of $\psi$, we first solve the following optimization problem. 
Minimize $E\left[\left(W_{M}(T)-\left(c_{M}+\lambda_{M}\right)\right)^{2}\right]$ over $\psi \in \Psi^{\pi}$, or equivalently, maximize $E[U$ $\left.\left(W_{M}(T)\right)\right]$ subject to $E\left[H(T) W_{M}(T)\right] \leq v^{\pi}(0)+w_{M}$, where $U(x)=-\left(x-\left(c_{M}+\lambda_{M}\right)\right)^{2}$ is a quadratic concave function of $x$ with inverse marginal $I(y)=\left(U^{\prime}\right)^{-1}(y)=-(1 / 2) y+$ $\left(c_{M}+\lambda_{M}\right)$. By martingale duality arguments, the necessary and sufficient conditions for the optimality are $\widehat{W}_{M}(T)=I(\hat{\alpha} H(T))$ for some $\hat{\alpha} \geq 0$ such that $v^{\pi}(0)+w_{M}=E[H(T)$ $\left.\widehat{W}_{M}(T)\right]$. So, the optimality condition is

$$
\widehat{W}_{M}(T)=-\frac{1}{2} \hat{\alpha} H(T)+\left(c_{M}+\lambda_{M}\right)
$$

so that $v^{\pi}(0)+w_{M}=-(1 / 2) \hat{\alpha} E\left[H^{2}(T)\right]+\left(c_{M}+\lambda_{M}\right) E[H(T)]$. Therefore, we obtain

$$
\begin{gathered}
\hat{\alpha}=2 \frac{\left(c_{M}+\lambda_{M}\right) E[H(T)]-\left(v^{\pi}(0)+w_{M}\right)}{E\left[H^{2}(T)\right]}, \\
\widehat{W}_{M}^{\pi}(T)=\frac{v^{\pi}(0)+w_{M}-\left(c_{M}+\lambda_{M}\right) E[H(T)]}{E\left[H^{2}(T)\right]} H(T)+\left(c_{M}+\lambda_{M}\right) .
\end{gathered}
$$

Before we find the optimal portfolio $\hat{\psi}=\widehat{\psi}\left(\lambda_{M}\right)$, we compute $v^{\pi}(t)$.

Lemma 2.14. The processes $v^{\pi}(t)=E_{t}^{0}\left[\int_{t}^{T} \gamma \phi(u, X(u)) \exp \left(-\int_{t}^{u} r(s) d s\right) d u\right]$, and $X(t)$ satisfies

$$
\begin{gathered}
v^{\pi}(t)=-X(t)\left(\exp \left(\int_{t}^{T} \gamma \rho(u) d u\right)-1\right), \quad 0 \leq t<T, \\
X(t)=-\frac{c \exp \left(\int_{0}^{T} \xi(u) d u\right)-w \exp \left(-\int_{0}^{T} r(u) d u\right)}{\exp \left(\int_{0}^{T} \xi(u) d u\right)-1} \exp \left(-\int_{t}^{T} r(u) d u\right)+W(t) .
\end{gathered}
$$

Proof. The identity (2.35) follows from (2.2) and (2.24). The discounted process $\bar{X}^{\hat{\pi}}$ satisfies the $\operatorname{SDE} d \bar{X}(t)=\bar{X}(t)\left[\gamma \rho(t) d t-\rho(t) \pi^{\prime} \sigma(t) d B^{0}(t)\right], 0<t \leq T$, with the unique solution

$$
\bar{X}(t)=x_{0} \exp \left(\int_{0}^{t}\left(\gamma \rho-\frac{1}{2} \xi\right)(u) d u-\int_{0}^{t} \rho \pi^{\prime} \sigma(u) d B^{0}(u)\right) .
$$

Hence, by (2.36) and Fubini's theorem,

$$
\begin{aligned}
v^{\pi}(t) & =-\int_{t}^{T} \gamma \rho(u) X(t) E_{t}^{0}\left[e^{\int_{t}^{u}(\gamma \rho-(1 / 2) \xi)(y) d y-\int_{t}^{u} \rho \pi^{\prime} \sigma d B^{0}(y)} d u\right] \\
& =-X(t) \int_{t}^{T} \gamma \rho(u) e^{\int_{t}^{u} \gamma \rho(s) d s} d u .
\end{aligned}
$$

The result then follows from the identity $\int_{t}^{T} \gamma \rho(u) e^{\int_{t}^{u} \gamma \rho(s) d s} d u=\left(e^{\int_{t}^{T} \gamma \rho(u) d u}-1\right)$.

As a corollary, we obtain the market value of the expected accumulated fees. 
Corollary 2.15. For $\pi \in \Pi_{f}$,

$$
v^{\pi}(0)=\frac{\exp \left(\int_{0}^{T} \gamma \rho(u) d u\right)-1}{1-\exp \left(-\int_{0}^{T} \xi(u) d u\right)}\left(c \exp \left(-\int_{0}^{T} r(u) d u\right)-w\right)
$$

Theorem 2.16. Let $\pi \in \Pi_{f}, \lambda_{M} \geq 0, \hat{\alpha}^{\pi}\left(\lambda_{M}\right) \geq 0$, and $\widehat{W}_{M}^{\pi}(T)$ be as in (2.33). Then the process $\bar{W}_{M}^{\pi}(t)$ defined by

$$
\bar{W}_{M}^{\pi}\left(t ; \lambda_{M}\right)=E_{t}^{0}\left[\widehat{W}_{M}^{\pi}\left(T ; \lambda_{M}\right)\right]-v^{\pi}(t)
$$

is the discounted wealth process for the manager that satisfies

$$
d \bar{W}_{M}(t)=\exp \left(-\int_{0}^{t} r(s) d s\right)\left(\gamma \phi d t+\psi_{M}^{\prime} \sigma d B^{0}(t)\right), \quad 0 \leq t \leq T,
$$

with $\bar{W}_{M}(0)=w_{M}>0$. Moreover, the optimal portfolio is

$$
\widehat{\psi}_{M}=\frac{\alpha}{2}\left(\sigma \sigma^{\prime}\right)^{-1} \sigma \theta(t) e^{\int_{t}^{T}\left(|\theta(s)|^{2}-2 r(s)\right) d s} H(t)-\rho(t) \pi(t) X(t)\left(e^{\int_{t}^{T} \gamma \rho(s) d s}-1\right) .
$$

Proof. Note that the expressions in (1.5) and (2.40) are equivalent with $\bar{W}_{M}(t)=W_{M}(t)$ $\exp \left(-\int_{0}^{t} r(s) d s\right)$. For $\psi_{M} \in \Psi,(1.5)$ implies that

$$
W_{M}(t) \exp \left(-\int_{0}^{t} r(s) d s\right)-\gamma \int_{0}^{t} \phi(u) \exp \left(-\int_{0}^{u} r(s) d s\right) d u
$$

is a square integrable martingale with respect to $P^{0}$. In particular, with $t=T$, we obtain the identity

$$
W_{M}(T) e^{-\int_{0}^{T} r(s) d s}-\int_{0}^{T} \gamma e^{-\int_{0}^{u} r(s) d s} \phi(u) d u=w_{M}+\int_{0}^{T} e^{-\int_{0}^{u} r(s) d s} \psi_{M}^{\prime} \sigma(u) d B^{0}(u),
$$

where, by Clark-Ocone formula, $\psi_{M}$ satisfies

$$
e^{-\int_{0}^{t} r(s) d s} \sigma^{\prime} \psi_{M}(t)=E_{t}^{0}\left[D_{t}\left(W_{M}(T) e^{-\int_{0}^{T} r(s) d s}-\int_{0}^{T} \gamma e^{-\int_{0}^{u} r(s) d s} \phi(u) d u\right)\right]
$$

and $D_{t}$ is the Malliavin derivative operator with respect to the Brownian motion $B^{0}$ (we refer the reader to the sources by Nualart [11] and Øksendal [12] for the technical aspects of Malliavin calculus). We have, by (2.31) and the chain rule of the Malliavin derivative,

$$
D_{t}\left(W_{M}(T)\right)=\frac{-\alpha}{2} D_{t}(H(T))=\frac{\alpha}{2} H(T) \theta(t)
$$

so that

$$
\begin{aligned}
E_{t}^{0}\left[D_{t}\left(W_{M}(T) e^{-\int_{0}^{T} r(s) d s}\right)\right] & =\frac{\alpha}{2} e^{-\int_{0}^{T} r(s) d s} \theta(t) E_{t}^{0}[H(T)] \\
& =\frac{\alpha}{2} e^{-\int_{0}^{T} 2 r(s) d s} \theta(t) e^{\int_{t}^{T}|\theta(s)|^{2} d s} Z_{0}(t)
\end{aligned}
$$


holds. Moreover, by using the properties of Malliavin derivative operator on integrals and applying Fubini's theorem,

$$
\begin{aligned}
& E_{t}^{0}\left[D_{t} \int_{0}^{T} \gamma e^{-\int_{0}^{u} r(s) d s} \phi(u) d u\right] \\
& \quad=E_{t}^{0}\left[-\gamma \int_{t}^{T} e^{-\int_{0}^{u} r(s) d s} \rho(u) D_{t} X(u) d u\right] \\
& =-\gamma e^{-\int_{0}^{t} r(s) d s}\left(-\rho \sigma^{\prime} \pi\right)(t) \int_{t}^{T} \rho(u) E_{t}^{0}\left[e^{-\int_{t}^{u} r(s) d s} X(u)\right] d u \\
& =\gamma e^{-\int_{0}^{t} r(s) d s} \rho \sigma^{\prime} \pi(t) X(t) \int_{t}^{T} \rho(u) e^{\int_{t}^{u} \gamma \rho(s) d s} d u \\
& =e^{-\int_{0}^{t} r(s) d s} \rho(t) \sigma^{\prime} \pi(t) X(t)\left(e^{\int_{t}^{T} \gamma \rho(s) d s}-1\right) .
\end{aligned}
$$

For $\pi \in \Pi_{f}$ and $\alpha \geq 0$, it follows from (2.44), (2.46), and (2.47) that

$$
\begin{aligned}
e^{-\int_{0}^{t} r(s) d s} \sigma^{\prime} \psi_{M}(t)= & \frac{\alpha}{2} e^{-\int_{0}^{T} 2 r(s) d s} \theta(t) e^{\int_{t}^{T}|\theta(s)|^{2} d s} Z_{0}(t) \\
& -e^{-\int_{0}^{t} r(s) d s} \rho(t) \sigma^{\prime} \pi(t) X(t)\left(e^{\int_{t}^{T} \gamma \rho(s) d s}-1\right)
\end{aligned}
$$

which implies that $\widehat{\psi}_{M}^{\alpha, \pi}$ coincides with the expression in (2.41).

Now, we will show that the process $\bar{W}_{M}^{\pi}$ defined in (2.39) satisfies (2.40), with $\psi=\widehat{\psi}_{M}^{\alpha, \pi}$ as in (2.41). By the identities (2.31), (2.34), (2.39), and (2.41), we get

$$
\begin{aligned}
d \widehat{W}_{M}^{\hat{\psi}_{M}^{\alpha, \pi}} & =d\left(E_{t}^{0}\left[\widehat{W}_{M}^{\hat{\psi}_{M}^{\alpha, \pi}}(T ; \lambda)\right]-v^{\pi}(t)\right) \\
& =-\frac{\alpha}{2} e^{-\int_{0}^{T} 2 r(s) d s} d\left(e^{\int_{t}^{T}|\theta(s)|^{2} d s} Z_{0}(T)\right)+d\left(\bar{X}(t)\left(e^{\int_{t}^{T} \gamma \rho(u) d u}-1\right)\right) \\
& =-\gamma \rho \bar{X}(t) d t+\left\{\frac{\alpha}{2} e^{\int_{t}^{T}\left(|\theta(s)|^{2}-2 r(s)\right) d s} e^{-\int_{0}^{t} r(s) d s} H(t)-\left(e^{\int_{t}^{T} \gamma \rho(u) d u}-1\right) \rho \pi^{\prime} \sigma \bar{X}(t)\right\} d B^{0}(t) .
\end{aligned}
$$

On the other hand, it is not difficult to check that the right-hand side of (2.40) coincides with (2.49) when $\psi=\widehat{\psi}_{M}^{\alpha, \pi}$.

Now, the optimal $\lambda_{M}$ in (2.30) maximizes $V_{M}^{\pi}\left(\lambda_{M}\right)=E\left[-U\left(\widehat{W}_{M}\left(T ; \lambda_{M}\right)\right)\right]-\lambda_{M}^{2}$ by Lagrange duality theorem, or equivalently, satisfies the condition $\left.E\left[\widehat{W}_{M}(T)\right)\right]=c_{M}$. Either way, we get

$$
\hat{\lambda}_{M}=\frac{c_{M} E[H(T)]^{2}-\left(w_{M}+v^{\pi}(0)\right) E[H(T)]}{E\left[H^{2}(T)\right]-E[H(T)]^{2}}
$$


which reduces to

$$
\frac{c_{M}-\left(w_{M}+v^{\pi}(0)\right) \exp \left(\int_{0}^{T} r(s) d s\right)}{\exp \left(\int_{0}^{T}|\theta(s)|^{2} d s\right)-1}=\exp \left(\int_{0}^{T} r(s) d s\right) \frac{\Delta-v^{\pi}(0)}{\exp \left(\int_{0}^{T}|\theta(s)|^{2} d s\right)-1}
$$

in the deterministic coefficients case, where

$$
\Delta=\exp \left(-\int_{0}^{T} r(s) d s\right) c_{M}-w_{M}
$$

Then, by (2.32) and (2.50), the corresponding Lagrange multiplier $\hat{\alpha}$ is

$$
\begin{aligned}
\hat{\alpha} & =2 \frac{c_{M} E[H(T)]-\left(w_{M}+v^{\pi}(0)\right)}{E\left[H^{2}(T)\right]-E[H(T)]^{2}} \\
& =2 \exp \left(\int_{0}^{T} r(s) d s\right) \frac{c_{M}-\left(w_{M}+v^{\pi}(0)\right) \exp \left(\int_{0}^{T} r(s) d s\right)}{\exp \left(\int_{0}^{T}|\theta(s)|^{2} d s\right)-1} \\
& =2 \exp \left(\int_{0}^{T} 2 r(s) d s\right) \frac{\Delta-v^{\pi}(0)}{\exp \left(\int_{0}^{T}|\theta(s)|^{2} d s\right)-1} .
\end{aligned}
$$

Corollary 2.17. For $\pi \in \Pi_{f}$ given, the optimal terminal wealth and the optimal portfolio of the manager are given by

$$
\begin{gathered}
\widehat{W}_{M}(T)=-\exp \left(\int_{0}^{T} r(s) d s\right) \frac{\Delta-v^{\pi}(0)}{\exp \left(\int_{0}^{T}|\theta(s)|^{2} d s\right)-1} Z_{0}(T)+\left(c_{M}+\lambda_{M}\right), \\
\widehat{\psi}_{M}^{\alpha, \pi}(t)=\frac{\Delta-v^{\pi}(0)}{\exp \left(\int_{0}^{T}|\theta(s)|^{2} d s\right)-1}\left(\sigma \sigma^{\prime}\right)^{-1} \sigma \theta Z_{0}(t) e^{\int_{t}^{T}|\theta(s)|^{2} d s} e^{\int_{0}^{t} r(s) d s}-\rho \pi(t) X(t)\left(e^{\int_{t}^{T} \gamma \rho(s) d s}-1\right),
\end{gathered}
$$

respectively.

2.3. Optimal fund portfolio. In the rest of this section, we assume $\pi \in \Pi_{f}$ and let $\Delta$ be as in (2.52). Then, the manager's optimal fund portfolio choice problem becomes minimizing the value function $V_{M}^{\pi}=E\left[-U\left(\widehat{W}_{M}\left(T ; \hat{\lambda}_{M}\right)\right)\right]-\widehat{\lambda}_{M}^{2}$ over $\Pi_{f}$ :

$$
V_{M} \triangleq \inf _{\pi \in \Pi_{f}} V_{M}^{\pi}=\inf _{\pi \in \Pi_{f}} E\left[\frac{\left(\hat{\alpha}^{\pi ; \hat{\lambda}_{M}}\right)^{2}}{4} H^{2}(T)\right]-\hat{\lambda}_{M}^{2}
$$


Now, by (2.50)-(2.55), straightforward computations show that

$$
\begin{aligned}
V_{M} & =\inf _{\pi \in \Pi} \frac{\left(\hat{\alpha}^{\pi ; \hat{\lambda}_{M}}\right)^{2}}{4} E\left[H^{2}(T)\right]-\hat{\lambda}_{M}^{2}=\inf _{\pi \in \Pi}\left\{\frac{1}{\operatorname{Var}(H(T))}\left(v^{\pi}(0)-\Delta\right)^{2}\right\} \\
& =\frac{\exp \left(2 \int_{0}^{T} r(s) d s\right)}{\exp \left(\int_{0}^{T}|\theta(s)|^{2} d s\right)-1} \inf _{\pi \in \Pi}\left\{\left(v^{\pi}(0)-\Delta\right)^{2}\right\},
\end{aligned}
$$

which attains the minimum value 0 when

$$
v^{\pi}(0)=\Delta=c_{M} \exp \left(-\int_{0}^{T} r(s) d s\right)-w_{M} .
$$

Hence, an optimal portfolio $\hat{\pi} \in \Pi_{f}$ is characterized by the condition (2.57) in the deterministic coefficients case if this identity is attained by some $\hat{\pi} \in \Pi_{f}$.

Remark 2.18. An optimal solution to (2.56) in the set $\Pi_{f}$ minimizes the cost functional $\left(v^{\pi}(0)-\Delta\right)^{2}$ which is not guaranteed to vanish. Numerical schemes (e.g., vector optimization techniques) would be used to obtain such a solution. Note also that the optimal $\alpha^{\pi}$ and $\lambda_{M}$ are zero when (2.57) holds. This implies that $W^{\hat{\pi}}(T)=c_{M}$, by $(2.31)$, meaning that the value function $V_{M}$ in (2.56) is zero. To summarize, we have the following.

Corollary 2.19. A fund weight process $\hat{\pi} \in \Pi_{f}$ is optimal if and only if

$$
\hat{\pi}=\arg \min _{\Pi_{f}}\left(v^{\pi}(0)-\Delta\right)^{2} .
$$

In particular, $v^{\hat{\pi}}(0)=\Delta$ if and only if $V_{M}=0$ (no risk for the manager). In this case, $\widehat{W}_{M}(T)=c_{M}$ with an optimal portfolio

$$
\widehat{\psi}_{M}^{\hat{\pi}}(t)=-\rho \hat{\pi} X(t)\left(\exp \left(\int_{t}^{T} \gamma \rho(s) d s\right)-1\right)=\phi(t, X(t)) \hat{\pi}(t)\left(\exp \left(\int_{t}^{T} \gamma \rho(s) d s\right)-1\right) .
$$

If $\exists \hat{\pi} \in \Pi_{f}$ satisfying (2.57), then by Corollary 2.15, we get the nonlinear equation

$$
\Delta=v^{\pi}(0)=\frac{\exp \left(\int_{0}^{T} \gamma \rho(u) d u\right)-1}{1-\exp \left(-\int_{0}^{T} \xi(u) d u\right)}\left[c \exp \left(-\int_{0}^{T} r(u) d u\right)-w\right] .
$$

Writing the dependence of $\xi(\cdot)$ and $\rho(\cdot)$ on $\pi$ in (2.60) explicitly, we are looking for a solution to the nonlinear equation

$$
\frac{\exp \left(\int_{0}^{T} \gamma\left(\left(\pi^{\prime} \sigma \theta-\gamma\right) /\left|\sigma^{\prime} \pi\right|^{2}\right)(u) d u\right)-1}{1-\exp \left(-\int_{0}^{T}\left(\left(\pi^{\prime} \sigma \theta-\gamma\right)^{2} /\left|\sigma^{\prime} \pi\right|^{2}\right)(u) d u\right)}\left[c \exp \left(-\int_{0}^{T} r(u) d u\right)-w\right]=\Delta
$$

for $\pi$. In general, numerical methods should be used to get a solution (among possibly multiple solutions) to this equation. We will show the existence of the solution in the constant coefficients case. 
Remark 2.20. Note that $v^{\pi}(0)$ is a linear increasing (decreasing, resp.) function of $c(w$, resp.), for any $t \in[0, T)$.

Lemma 2.21. Assume that all the parameters of the model are constant and $n=1$. Define $v(\pi) \triangleq v^{\pi}(0)$ as a function of $\pi \in \mathbb{R}$. Then $v(\pi)$ satisfies the following:

(a) $v$ is a continuous function of $\pi \in(\gamma /(\mu-r), \infty)=\{\pi \in \mathbb{R}: \rho>0\}$,

(b) $\lim _{\pi \rightarrow(\gamma /(\mu-r))^{+}} v(\pi)=\infty, \lim _{\pi \rightarrow \infty} v(\pi)=0$,

(c) $v$ is strictly positive on $(\gamma /(\mu-r), \infty)$,

(d) $d v(\pi) / d \pi<0$ (a strictly decreasing function of $\pi$ ) on $[2 \gamma /(\mu-r), \infty$ ).

Proof. The claims (a)-(c) are clear. For (d), see Appendix C.

Remark 2.22. Lemma 2.21 assures that for any $\Delta>0, \exists \pi \in(\gamma /(\mu-r), \infty)$ with $v(\pi)=$ $\Delta$ when all the parameters are constant. Hence there is always a solution to (2.61) in $(\gamma /(\mu-r), \infty)$, for $n=1$. If $v$ is also monotone in $(\gamma /(\mu-r), 2 \gamma /(\mu-r))$, this solution is unique.

Theorem 2.23. Let $n \geq 1$ be given and let all the parameters of the model be constant. Then, for any $\Delta>0$, there exist at least $n$ constant portfolio weights in $\Pi_{f}$ such that (2.61) holds.

Proof. Let $\Delta>0$ be given. When $n=1$, the existence of a solution in the interval $(\gamma /(\mu-r)$, $\infty$ ) follows from Remark 2.22. When $n>1$, the problem can be reduced to the onedimensional case as follows: for each $i=1, \ldots, n$, consider weights of the form $\pi^{i}=\left(\pi^{i}(1)\right.$, $\left.\ldots, \pi^{i}(n)\right)^{\prime}$, where $\pi^{i}(i) \in\left(\gamma /\left(\mu_{i}-r\right), \infty\right)$, and $\pi^{i}(j)=0$ if $j \neq i$. Then we have

$$
\begin{aligned}
v\left(\pi^{i}\right) & =\frac{e^{\gamma\left(\left(\pi^{i} \sigma \theta-\gamma\right) /\left|\sigma^{\prime} \pi^{i}\right|^{2}\right) T}-1}{1-e^{-\left(\left(\pi^{i} \sigma \theta-\gamma\right)^{2} /\left|\sigma^{\prime} \pi^{i}\right|^{2}\right) T}}\left(c e^{-r T}-w\right) \\
& =\frac{e^{\gamma\left(\pi^{i}(i)(\mu(i)-r) /\left|\sigma_{i}\right|^{2}\left(\pi^{i}(i)\right)^{2}\right) T}-1}{1-e^{-\left(\pi^{i}(i)(\mu(i)-r) /\left|\sigma_{i}\right|\left(\pi^{i}(i)\right)\right)^{2} T}}\left(c e^{-r T}-w\right),
\end{aligned}
$$

where $\sigma_{i}$ is the $i$ th row of the matrix $\sigma$. Since $\mu(i)-r>0$ and $\left|\sigma_{i}\right|>0$, for each $i=1, \ldots, n$, (2.61) reduces to the one-dimensional problem

$$
f(p) \triangleq \frac{e^{\gamma\left(p(\mu(i)-r) /\left|\sigma_{i}\right|^{2} p^{2}\right) T}-1}{1-e^{-\left(p(\mu(i)-r) /\left|\sigma_{i}\right| p\right)^{2} T}}\left(c e^{-r T}-w\right)=\Delta,
$$

by (2.62). It is easy to see that $v\left(\pi^{i}\right)=f\left(\pi^{i}(i)\right)$ and $f$ satisfies the identities in Lemma 2.21. So there is a solution $\hat{\pi}^{i}(i) \in\left(\gamma /\left(\mu_{i}-r\right), \infty\right)$ of (2.63), for each $i$. This implies that there are at least $n$ solutions of (2.61).

Remark 2.24. If the manager's target wealth level $c_{M}$ is too large, then any solution $\hat{\pi}^{i}(i)$ may be close to $\gamma /\left(\mu_{i}-r\right)$ with a relatively large value of $v\left(\hat{\pi}^{i}(i)\right)$, by Lemma 2.21(b). Moreover, if the manager had an increasing concave utility function, an optimal fund weight $\hat{\pi}$ maximizes $v^{\pi}(0)$, as in [6], even though the investor has mean-variance preferences. However, $\hat{\pi}$ still does not maximize after-the-fees Sharpe ratio $\rho$. Indeed, an optimal $\hat{\pi}$ could be very close to $\gamma /(\mu-r)$ or may not exist in this setup (e.g., when $v(\pi)$ is 
monotonically decreasing). So the "practical" constant fund weights would be bounded away from $\gamma /(\mu-r)$ satisfying $\rho^{\pi}>L>0$, for some $L$.

Example 2.25. Let $w=100, c=115, r=0.05, \mu=0.20, \sigma=0.35, \gamma=0.015, T=1, c_{M}=$ 235 , and $w_{M}=200$. Then $\pi$ is a root of the function

$$
\begin{aligned}
F(p) & =v(p)-\left(c_{M} \exp \left(-\int_{0}^{T} r(s) d s\right)-w_{M}\right) \\
& =\left(e^{-0.05} 115-100\right) \frac{e^{(0.015)\left(((0.15) p-0.015) /(0.35 p)^{2}\right)}-1}{1-e^{-(0.15 p-0.015)^{2} /(0.35 p)^{2}}}-\left(235 e^{-0.05}-200\right),
\end{aligned}
$$

$v(p)$ is strictly decreasing on $(\gamma /(\mu-r), \infty)=(0.1, \infty)$ and so is $F(p)$, with a unique zero at $p^{*} \simeq 0.1409787$ (see Figure 2.1). Recall that the risk for the manager is zero. The risk for the investor is

$$
\Gamma\left(p^{*}\right)=\frac{\left(115-100 e^{0.05}\right)^{2}}{e^{\left(0.15 p^{*}-0.015\right)^{2} /\left(0.35 p^{*}\right)^{2}}-1} \simeq 6232.4513
$$

which is quite larger than the minimum variance $\left(115-100 e^{0.05}\right)^{2} /\left(\exp \left((0.15)^{2} /(0.35)^{2}\right)-\right.$ $1)=483.45$ if the investor had direct access to the risky asset, as indicated in Remark 2.10. It is essential that the manager invests in the stock market for her own portfolio to achieve zero overall risk. Straightforward computations show that (see Appendix C) the manager's wealth process with $\psi=0$ and $W_{M}(0)=w_{M}>0$ is given by

$$
W_{M}^{p}(1)=e^{r}\left(w_{M}+\gamma \rho \frac{c e^{-r}-w}{1-e^{-\xi}} \int_{0}^{1} e^{-(3 / 2) \xi t-\rho p \sigma B(t)} d t\right)
$$

so that

$$
E\left[W_{M}^{p}(1)\right]=e^{r}\left(w_{M}+\gamma \frac{c e^{-r}-w}{p(\mu-r)-\gamma}\right) .
$$

The $p$ value that solves for $E\left[W_{M}^{p}(1)\right]=235$ is $p \simeq 0.1399<\hat{p}$. By Monte Carlo simulations (100000 simulated paths) and uniform Euler discretization (with 250 points on $[0,1])$, the variance $\operatorname{Var}\left(W_{M}^{p}(1)\right)$ is computed approximately as $\operatorname{Var}\left(W_{M}^{p}(1)\right) \simeq 3.0561>$ 0 .

Example 2.26. Now, consider three-dimensional generalization of Example 2.25 with a vector of appreciation rates $\mu=\left[\begin{array}{lll}0.20 & 0.15 & 0.18\end{array}\right]^{\prime}$ and a diagonal volatility matrix $\sigma$ with entries $\sigma_{11}=0.35, \sigma_{22}=0.25$, and $\sigma_{33}=0.30$. Then, by Theorem 2.23, the following fund portfolio weights result in zero risk for the manager: $\pi^{1}=\left[\begin{array}{lll}p_{1} & 0 & 0\end{array}\right]^{\prime}$, $\pi^{2}=\left[\begin{array}{lll}0 & p_{2} & 0\end{array}\right]^{\prime}, \pi^{3}=\left[\begin{array}{lll}0 & 0 & p_{3}\end{array}\right]^{\prime}$ with $p_{1}=0.1409787, p_{2}=0.2112526$, and $p_{3}=$ 0.1626966 . Actually, there are many other solutions which could be obtained by assigning arbitrary weights for one or two components and computing the remaining one(s) numerically. For instance, if the manager is seeking an optimal portfolio weight of the form $\pi=\left[\begin{array}{lll}p & -0.1 & 0.05\end{array}\right]^{\prime}$, then $p=0.16385$ is the proportion of the first stock of such a fund portfolio. 


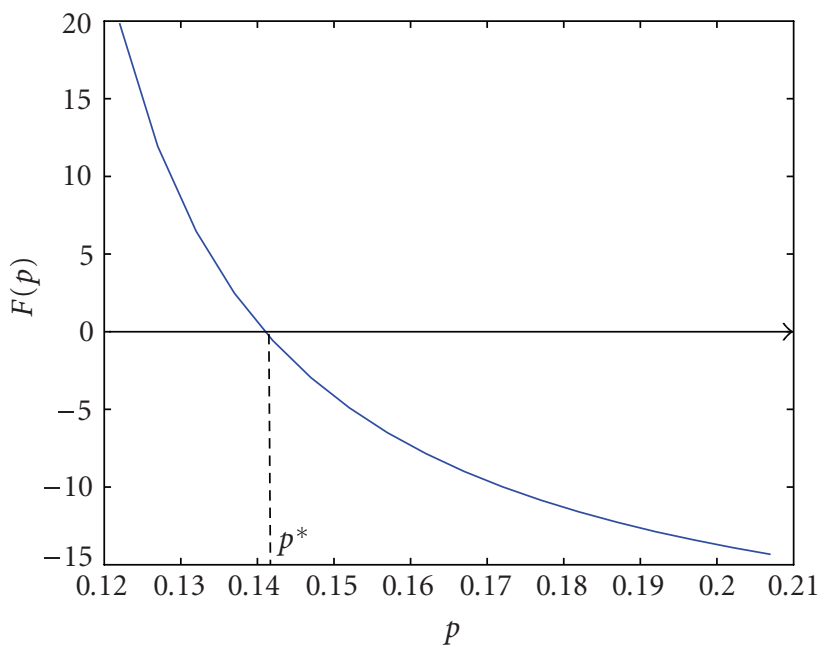

Figure 2.1. The graph of the nonlinear function $F$ for Example 2.25.

2.4. Financial interpretation, extension to multiple managers. In a single manager framework, the manager has full access to the stock market. By observing the response of the investor to the possible fund strategies, she can choose an optimal fund portfolio from her perspective. By (2.59), for each dollar that investor keeps in the fund at time $t$ (the dollar proportion invested in each stock in the fund is $\pi_{i}(t)$ ), she invests $\pi_{i}(t)\left(\exp \left(\gamma \int_{t}^{T} \rho(s) d s-1\right)\right)$ dollars for each stock $i$, for $i=1, \ldots, n$, in her own portfolio. Note that, provided $\pi(t)$ is feasible for the investor, some of the $\pi_{i}$ 's are allowed to be negative indicating short selling of such stocks. In the constant coefficients case, she can find a constant feasible fund portfolio weight $\pi$ and a hedging portfolio $\psi$ for her own investment so that the target wealth $c_{M}$ is always achieved with zero variance.

The set of feasible fund weights depends on the fee rate $\gamma$. Therefore, the optimal $\pi$ depends on $\gamma$, too. Our numerical simulations for Example 2.25 indicates that the optimal fund portfolio proportion $\hat{\pi}(\gamma)$ is a linear increasing function of $\gamma$ in the one-dimensional constant coefficients case, as in Figure 2.2. However, the value function $\Gamma(\hat{\pi}(\gamma), \gamma)$ stays constant (independent of $\gamma>0$ and is equal to 6232.4513) for the optimal strategies, similar to the results in [6]. Therefore, it does not matter which agent decides the fee rate. For a heuristic discussion of these results, see also Appendix C.

In general, the risk for the investor increases with $c$, by Remark 2.12. In the onedimensional constant coefficients case, it is an increasing function of $c_{M}$ if $v(p)$ is monotonically decreasing on $(\gamma /(\mu-r), \infty)$, by Remarks 2.12 and 2.22 and Lemma 2.21. Moreover, the $c$ and $c_{M}$ values compete with each other. So, when the manager increases her target level $c_{M}$, the investor should decrease $c$ to maintain the same level of the risk. Otherwise, he will face a higher level of variance due to a decrease in the optimal fund portfolio weight $\pi$. In this case, he would be paying management fees for a fund primarily consisting of the risk-free bond. This fact implies that in a single manager framework, the 


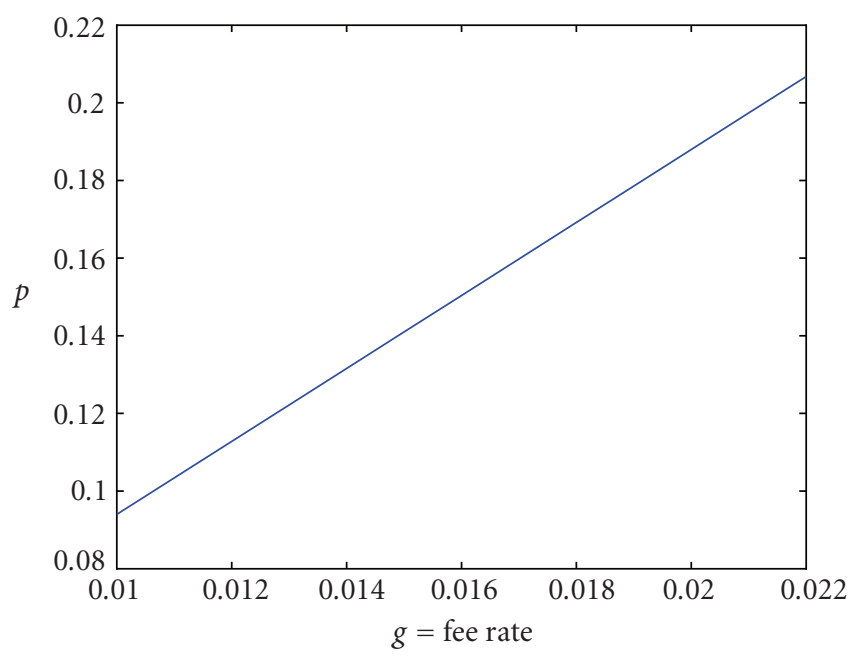

Figure 2.2. The optimal fund portfolio weight versus fee rate.

manager can take advantage of the investor as being the strategic leader and the only service provider. However, the case of multiple managers would allow the investor to benefit from a competition among various managers using the identity (2.25). This fact can be expressed by the following general result in a multidimensional setting.

Theorem 2.27. Consider $K$ managers in the financial market model given in Section 1.1 and let $\hat{\pi}_{i}$ be an $n$-dimensional feasible optimal fund portfolio weight for the manager $i$, $i=1, \ldots, K$. Then the investor will choose the manager $j$ to minimize his risk if

$$
\int_{0}^{T} \xi^{\hat{\pi}_{j}}(s) d s=\max _{1 \leq i \leq K}\left\{\int_{0}^{T} \xi^{\hat{\pi}_{i}}(s) d s\right\} .
$$

Proof. For any feasible weight $\pi$, the risk $\operatorname{Var}(W(T))$ of the investor is inversely proportional to the quantity $\int_{0}^{T} \xi(s) d s$, by (2.25). Hence minimizing $\operatorname{Var}(W(T))$ amounts to maximizing $\int_{0}^{T} \xi(s) d s$ over $\pi$.

When the fund portfolio proportions $\pi$ are further restricted to the ones satisfying $\rho(\cdot)>L>0$, the risk for the investor is bounded above as described in Remark 2.24. Although this setup can be generalized to include managers with increasing concave utility functions, those managers would not contribute to the competition due to a lower level of $\int_{0}^{T} \xi(s) d s$. We have then the following result in the one-dimensional case.

THeOREM 2.28. Consider a financial market with one risky asset and one investor whose target terminal wealth is $c>w e^{r T}$. Assume also that

(i) all the parameters are constant and are such that the function $v$ given by (2.61) is monotone decreasing on $(\gamma /(\mu-r), \infty)$; 
(ii) there are $K$ managers with mean-variance preferences and with target terminal wealth levels $c_{M_{i}}$ such that $\Delta_{M_{i}}=c_{M_{i}} e^{-r T}-w_{M_{i}}>0$, for $i=1, \ldots, K$, and $\Delta_{M_{1}} \geq$ $\Delta_{M_{2}} \geq \cdots \geq \Delta_{M_{K}}$ (known by the investor);

(iii) there is one manager $((K+1)$ st one $)$ with increasing concave utility function.

Then each manager $i=1, \ldots, K$ has a unique optimal fund weight $\hat{\pi}_{i}$, with $\gamma /(\mu-r)<\hat{\pi}_{1} \leq$ $\hat{\pi}_{2} \leq \cdots \leq \hat{\pi}_{K}$, and the investor will choose Kth manager to minimize his risk. No optimal weight $\hat{\pi}_{K+1}$ exists for the manager $K+1$ on $(\gamma /(\mu-r), \infty)$. However, $\hat{\pi}_{K+1}=\gamma /(\mu-r)+\epsilon$, whenever $\Pi_{f}=[\gamma /(\mu-r)+\epsilon, D]$, where $\epsilon$ and $D$ are positive constants with $D>\gamma /(\mu-r)+\epsilon$.

\section{Appendices}

\section{A. The viscosity solution to the HJB equation (2.7)}

Lemma A.1. For $v \geq 0$, define the function $l(v)=\alpha v+(1 / 2) \beta v^{2}$ with $\beta>0$. Then $l(v)$ is minimized at $\hat{v}=(-\alpha / \beta)^{+}$with the minimum value $l(\hat{v})=-\left(\alpha^{2} / 2 \beta\right) 1_{\{\hat{v}>0\}}$.

By Lemma A.1, the expression $\inf _{\phi \geq 0}\left\{\phi\left(\pi^{\prime} \sigma \theta-\gamma\right) v_{x}+(1 / 2) \phi^{2}\left|\sigma^{\prime} \pi\right|^{2} v_{x x}\right\}$ is minimized at $\phi=\left(-\left(\left(\pi^{\prime} \sigma \theta-\gamma\right) /\left|\sigma^{\prime} \pi\right|^{2}\right)\left(v_{x} / v_{x x}\right)\right)^{+}$that indeed characterizes an optimal portfolio for the investor when $\pi \in \Pi_{f}$, as shown in the proof below.

Proof of Proposition 2.3. We first consider the quadratic trial function

$$
v(t, x)= \begin{cases}a(t) x^{2}, & (t, x) \in R_{1}, \\ A(t) x^{2}, & (t, x) \in R_{2},\end{cases}
$$

with $v(T, x)=x^{2}$ as a candidate for a viscosity solution of (2.7), where the sets

$$
\begin{aligned}
& R_{1}=\{(t, x): x \rho(t) \leq 0,0 \leq t<T\}, \\
& R_{2}=\{(t, x): x \rho(t)>0,0 \leq t<T\}
\end{aligned}
$$

are the regions, where $\phi$ is nonnegative and negative, respectively; whereas $R_{3}$ is the boundary between $R_{1}$ and $R_{2}: R_{3}=\{(t, x): x \rho(t)=0\}$.

Plugging the trial function (A.1) into the HJB PDE, we obtain the linear ODEs

$$
\begin{gathered}
\dot{a}+(2 r-\xi) a=0, \quad a(T)=1, \\
\dot{A}+2 r A=0, \quad A(T)=1,
\end{gathered}
$$

with the solutions as in (2.8): $A(t)=\exp \left(\int_{t}^{T} 2 r(s) d s\right), a(t)=A(t) \exp \left(\int_{t}^{T}(-\xi)(s) d s\right)$. The sets $R_{1}$ and $R_{2}$ can be written as $R_{1}=R_{1,1} \cup R_{1,2}$ and $R_{2}=R_{2,1} \cup R_{2,2}$, where

$$
\begin{aligned}
& R_{1,1}=\{(t, x): x \leq 0, \rho(t)>0,0 \leq t<T\}, \\
& R_{1,2}=\{(t, x): x>0, \rho(t) \leq 0,0 \leq t<T\}, \\
& R_{2,1}=\{(t, x): x>0, \rho(t)>0,0 \leq t<T\}, \\
& R_{2,2}=\{(t, x): x<0, \rho(t)<0,0 \leq t<T\} .
\end{aligned}
$$


Then $a(t) x^{2}$ and $A(t) x^{2}$ satisfy $a(t) x^{2} \in C^{1,2}\left(R_{1}\right), A(t) x^{2} \in C^{1,2}\left(R_{2,1}\right) \cap C^{1,2}\left(R_{2,2}\right)$. The $\mathrm{HJB}$ equation in $R_{1}$ is

$$
\dot{a} x^{2}+r x(2 a x)+\inf _{\phi \geq 0}\left\{\phi\left(\pi^{\prime} \sigma \theta-\gamma\right)(2 a x)+\frac{1}{2} \phi^{2}\left|\sigma^{\prime} \pi\right|^{2}(2 a)\right\}=0,
$$

where $\left\{\phi\left(\pi^{\prime} \sigma \theta-\gamma\right)(2 a x)+\phi^{2}\left|\sigma^{\prime} \pi\right|^{2} a\right\}$ is minimized at $\phi=-\left(\left(\pi^{\prime} \sigma \theta-\gamma\right) /\left|\sigma^{\prime} \pi\right|^{2}\right)(t) x=$ $-\rho(t) x$ with the minimum value $-a\left(\left(\pi^{\prime} \sigma \theta-\gamma\right)^{2} /\left|\sigma^{\prime} \pi\right|^{2}\right)(t) x^{2}=a \xi(t) x^{2}$. So, the left-hand side of (A.5) is $(\dot{a}+2 r-\xi) x^{2}$ which vanishes by (A.3). Hence, the function $v^{1}(t, x) \triangleq$ $a(t) x^{2}$ is a smooth solution to the HJB equation in $R_{1}$. Similarly, it is easy to see that the function $v^{2}(t, x) \triangleq A(t) x^{2}$ is a smooth solution for the HJB equation in $R_{2,1}$ and $R_{2,2}$, separately.

For the boundary region between $R_{1}$ and $R_{2}$, we have $x \rho(t)=0$. Substituting $x=0$ in $v^{1}$ and $v^{2}$ and noting the boundary conditions $v^{1}(T)=0=v^{2}(T)$, we get, as a function of $t, v^{1}(t)=0=v^{2}(t)$ and therefore $v_{t}(t, x)=0$ and $v_{x}^{1}(t, x)=0=v_{x}^{2}(t, x)$ on the boundary between $R_{1,1}$ and $R_{2,2}$ and hence between $R_{2,1}$ and $R_{1,2}$ showing that $v \in C^{1,1}$ on these boundaries. However, $v \notin C^{1,2}$ since $v_{x x}^{1}=2 a \neq 2 A=v_{x x}^{2}$. A similar result can be obtained on the boundary region with $\rho(t)=0$, that is, between the regions $R_{2,1}$ and $R_{1,1}$ and regions $R_{2,2}$ and $R_{1,2}$. It is not difficult to prove that $v$ is both a viscosity subsolution and a viscosity supersolution of the HJB equation satisfying the boundary condition $v(T, x)=x^{2}$. Hence $v(t, x)$ defined by (A.1) with coefficients as in (2.8) is a viscosity solution to (2.7) when $\pi \in \Pi_{f}$. Moreover, the SDE (2.3) with $\phi$ given in Proposition 2.3 is a linear SDE with a unique strong solution. The proof of Proposition 2.3 is then follows from verification theorems for viscosity solutions. See, for example, [5, 14]. See also [9] for a similar application with no shorting constraints.

\section{B. Proof of Proposition 2.8}

In this part, we discuss the optimization problem

$$
V^{\pi}(s, x(\lambda))=\inf _{\phi>0} J^{\pi}(s, x(\lambda) ; \phi)
$$

for fixed $\lambda \geq 0$, using the martingale duality approach. Given the quadratic utility function $U(x)=-x^{2}$ and $\pi \in \Pi_{f}$ with $\rho^{\pi}(\cdot)>0$, we have

$$
V^{\pi}\left(0, x_{0}\right)=-\sup _{\phi>0} E\left[U\left(X_{T}^{\pi, \phi}\right)\right]
$$

Now, we introduce the following processes: $\widetilde{B}(t)=B(t)+\int_{0}^{t} \tilde{\theta}(s) d s$ and $\tilde{Z}(t)=\exp (-$ $\left.(1 / 2) \int_{0}^{t}|\tilde{\theta}(s)|^{2} d s-\int_{0}^{t} \tilde{\theta}(s) d B(s)\right)$, where $\tilde{\theta}(t)=\rho \sigma^{\prime} \pi(t)=\left(\left(\pi^{\prime} \sigma \theta-\gamma\right) /\left|\sigma^{\prime} \pi\right|^{2}\right) \sigma^{\prime} \pi(t)$. We also define an equivalent probability measure $\widetilde{P}$ as $\widetilde{P}(\cdot) \triangleq E[1(\cdot) \tilde{Z}(T)]$. Then by checking the Novikov condition (see [8], e.g.) for both $\widetilde{B}$ and $\bar{X}$, it is easy to see that when $\pi \in \Pi_{f}$ with $\rho^{\pi}(\cdot)>0, \widetilde{B}$ is an $n$-dimensional Brownian motion under $\widetilde{P}, \widetilde{Z}$ is a martingale under $P$, and the discounted process $\bar{X}(\cdot) \triangleq X(\cdot) / S_{0}(\cdot)$ is a martingale with respect to $(\tilde{B}, \tilde{P})$ : $d \bar{X}(t)=\bar{\phi} \pi^{\prime} \sigma d \widetilde{B}(t), 0<t \leq T$, with $\bar{X}(0)=x<0$, and $\bar{\phi}(t)=\phi(t) / S_{0}(t)$. 
Now, by (B.2) and the fact that $\bar{X}$ is a $(\widetilde{B}, \widetilde{P})$ martingale, it suffices to solve the following optimization problem:

$$
\text { maximize } E\left[U\left(X_{T}\right)\right] \quad \text { subject to } \tilde{E}[\bar{X}(T)]=x_{0} \text { over } u \text {. }
$$

Let $I(\cdot)$ be the inverse of the marginal utility function: $I(y) \triangleq(d U / d y)^{-1}(y)=-y / 2$. Then a standard application of martingale duality to utility maximization yields that the optimal terminal condition is given by

$$
\hat{X}(T)=I\left(\frac{\hat{\alpha} \tilde{Z}(T)}{S_{0}(T)}\right),
$$

where $\hat{\alpha}$ is a Lagrange multiplier such that the static terminal condition holds:

$$
x_{0}=\widetilde{E}\left[\frac{\hat{X}(T)}{S_{0}(T)}\right]=E\left[I\left(\frac{\hat{\alpha} \tilde{Z}(T)}{S_{0}(T)}\right) \frac{\tilde{Z}(T)}{S_{0}(T)}\right]=-\hat{\alpha} E\left[\frac{\tilde{Z}^{2}(T)}{2 S_{0}^{2}(T)}\right] .
$$

Since $E\left[\tilde{Z}^{2}(T)\right]=\exp \left(\int_{0}^{T}|\tilde{\theta}(s)|^{2} d s\right)=\exp \left(\int_{0}^{T} \xi(s) d s\right)$, we get $\hat{\alpha}=-2 x_{0} \exp \left(\int_{0}^{T}(2 r-\xi)(s) d s\right)$ $>0$ from (B.5). Then (B.4) implies that

$$
\begin{aligned}
\hat{X}(T) & =x_{0} \exp \left(\int_{0}^{T}(2 r-\xi)(s) d s\right) \frac{\tilde{Z}(T)}{S_{0}(T)} \\
& =x_{0} \exp \left(\int_{0}^{T}\left(\frac{r-3 \xi}{2}\right)(s) d s-\int_{0}^{T} \tilde{\theta}(s) d B(s)\right) .
\end{aligned}
$$

Proof of Proposition 2.8. By the duality arguments, $\hat{X}(T)$ given by (B.6) is optimal with

$$
\sup _{\phi} E\left[U\left(X_{T}^{\pi, \phi}\right)\right]=E\left[-\hat{X}^{2}(T)\right]=-x_{0}^{2} \exp \left(\int_{0}^{T}(2 r-\xi)(s) d s\right)
$$

so that $V(0, x)=x_{0}^{2} \exp \left(\int_{0}^{T}(2 r-\xi)(s) d s\right)$. Now, by direct verification, (2.3) with $X_{0}=x_{0}$ and $\hat{\phi}(t)$ as in (2.16) becomes $d X_{t}=X_{t}\left[(r-\xi) d t-\rho \pi^{\prime} \sigma d B_{t}\right]$ with the unique solution

$$
X_{t}^{\hat{\phi}}=x_{0} \exp \left(\int_{0}^{t}\left(\frac{r-3 \xi}{2}\right)(s) d s-\int_{0}^{t} \tilde{\theta}(s) d B_{s}\right)
$$

and the terminal value $X_{T}=\hat{X}_{T}$. Clearly, $X^{\hat{\phi}}<0$ and $\hat{\phi}>0$ a.s. on $[0, T]$ and $\hat{\phi} \in \mathcal{U}$.

\section{Proofs of some lemmas and claims}

(i) Computations in Example 2.25. The manager's wealth process with $\psi=0$ satisfies

$$
d W_{M}(t)=\left(W_{M} r+\gamma \phi\right) d t=\left(W_{M} r-\gamma \rho X(t)\right) d t, \quad 0<t \leq 1,
$$

so that

$$
W_{M}^{p}(1)=e^{r}\left(w_{M}-\int_{0}^{1} \gamma \rho \bar{X}(t) d t\right)=e^{r}\left(w_{M}+\gamma \rho \frac{c e^{-r}-w}{1-e^{-\xi}} \int_{0}^{1} e^{-(3 / 2) \xi t-\rho p \sigma B(t)} d t\right) .
$$


Then, with $\rho=(\mu-r) / \sigma^{2} p-\left(\gamma / \sigma^{2} p^{2}\right)$,

$$
\begin{aligned}
E\left[W_{M}(1)\right] & =e^{r}\left(w_{M}-\gamma \rho \frac{w e^{\xi}-c e^{\xi-r}}{e^{\xi}-1} \int_{0}^{1} E\left[e^{-(3 / 2) \xi t-p \rho \sigma B(t)}\right] d t\right) \\
& =e^{r}\left(w_{M}+\gamma \rho \frac{c e^{\xi-r}-w e^{\xi}}{e^{\xi}-1} \int_{0}^{1} e^{-\xi t} d t\right)=e^{r}\left(w_{M}+\gamma \frac{c e^{-r}-w}{\hat{p}(\mu-r)-\gamma}\right) .
\end{aligned}
$$

(ii) Proof of Lemma 2.21(d). The function $v(p)$ can be written as $v(p)=f(p)\left(c e^{-r T}-w\right)$ with $f(p)=\left(e^{\gamma \rho(p) T}-1\right) /\left(1-e^{-\xi(p) T}\right)$ and $c e^{-r T}-w>0$. We have

$$
\frac{d f(p)}{d p}=T \frac{\gamma(d \rho(p) / d p) e^{\gamma \rho(p) T}\left(1-e^{-\xi(p) T}\right)-\left(e^{\gamma \rho(p) T}-1\right)(d \xi(p) / d p)}{\left(1-e^{-\xi(p) T}\right)^{2}} .
$$

Note that $\rho>0$ and $\xi>0$ on $(\gamma /(\mu-r), \infty), d \rho(p) / d p \leq 0$, and $d \xi(p) / d p>0$ on $[2 \gamma /(\mu-r)$, $\infty)$. So the numerator of $d f(p) / d p$ is always negative on $[2 \gamma /(\mu-r), \infty)$ and $v$ is strictly decreasing there.

(iii) Heuristics for financial interpretation. For small values of $\gamma>0$, the function $f(p)$ can be reasonably approximated by using the first-order Taylor approximation as $f(p) \simeq$ $\gamma \rho(p) / \xi(p)=\gamma /(p \sigma \theta-\gamma)$. Hence $v(p) \simeq\left(c e^{-r T}-w\right)(\gamma /(p \sigma \theta-\gamma))$ and the equation $\left(c e^{-r T}-w\right)(\gamma /(p \sigma \theta-\gamma))=\Delta$ has a unique solution $p^{*}=\gamma\left(c e^{-r T}-w\right) / \Delta>0$ which is increasing with $\gamma$. Moreover, the value function $\Gamma$ in Remark 2.12 is

$$
\Gamma\left(p^{*}\right)=\frac{\left(c e^{-r T}-w\right)^{2}}{e^{\xi\left(p^{*}\right)}-1} \simeq \frac{e^{2 r}\left(c e^{-r T}-w\right)^{2}}{\xi\left(p^{*}\right)}=\frac{e^{2 r}\left(c e^{-r}-w+\Delta\right)^{2}}{\theta^{2}}
$$

which is independent of $\gamma>0$. For Example 2.25, these approximations yield $p^{*} \simeq 0.1399$ and $\Gamma\left(p^{*}\right) \simeq 6524.9$.

\section{Acknowledgments}

The author would like to thank Jaksa Cvitanic, Henry Schellhorn, and the referee(s) for their valuable suggestions and corrections.

\section{References}

[1] T. R. Bielecki, H. Jin, S. R. Pliska, and X. Y. Zhou, Continuous-time mean-variance portfolio selection with bankruptcy prohibition, Mathematical Finance 15 (2005), no. 2, 213-244.

[2] C. Cetin, Backward stochastic differential equations with quadratic growth and their applications, Ph.D. Dissertation, University of Southern California, California, 2005, http://web.whittier.edu/ ccetin.

[3] J. Cvitanić and I. Karatzas, Convex duality in constrained portfolio optimization, The Annals of Applied Probability 2 (1992), no. 4, 767-818.

[4] J. Cvitanić, X. Wan, and J. Zhang, Optimal contracts in continuous-time models, to appear in Journal of Applied Mathematics and Stochastic Analysis.

[5] W. H. Fleming and H. M. Soner, Controlled Markov Processes and Viscosity Solutions, Applications of Mathematics (New York), vol. 25, Springer, New York, 1993. 
[6] J. Hugonnier and R. Kaniel, Mutual fund portfolio choice in the presence of dynamic flows, preprint, $2006 \mathrm{http}: / /$ faculty.fuqua.duke.edu/ rkaniel/webpage/.

[7] I. Karatzas and S. E. Shreve, Methods of Mathematical Finance, Applications of Mathematics (New York), vol. 39, Springer, New York, 1998.

[8]__ Brownian Motion and Stochastic Calculus, Springer, New York, 1999.

[9] X. Li, X. Y. Zhou, and A. E. B. Lim, Dynamic mean-variance portfolio selection with no-shorting constraints, SIAM Journal on Control and Optimization 40 (2002), no. 5, 1540-1555.

[10] A. E. B. Lim, Quadratic hedging and mean-variance portfolio selection with random parameters in an incomplete market, Mathematics of Operations Research 29 (2004), no. 1, 132-161.

[11] D. Nualart, The Malliavin Calculus and Related Topics, Probability and Its Applications (New York), Springer, New York, 1995.

[12] B. Øksendal, An Introduction to Malliavin Calculus with Applications to Economics, Lecture Notes, Norwegian School of Economics and Business Administration, Norway, 1997.

[13] H. Ou-Yang, Optimal contracts in a continuous-time delegated portfolio management problem, The Review of Financial Studies 16 (2003), no. 1, 173-208.

[14] X. Y. Zhou, J. Yong, and X. Li, Stochastic verification theorems within the framework of viscosity solutions, SIAM Journal on Control and Optimization 35 (1997), no. 1, 243-253.

Coskun Cetin: Department of Mathematics, Whittier College, Whittier, CA 90608-0634, USA

E-mail address: ccetin@whittier.edu 


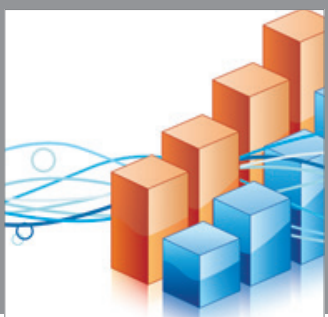

Advances in

Operations Research

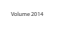

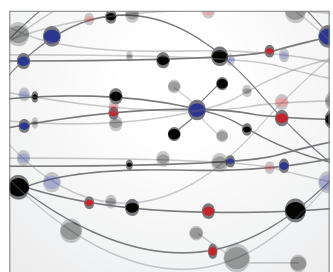

\section{The Scientific} World Journal
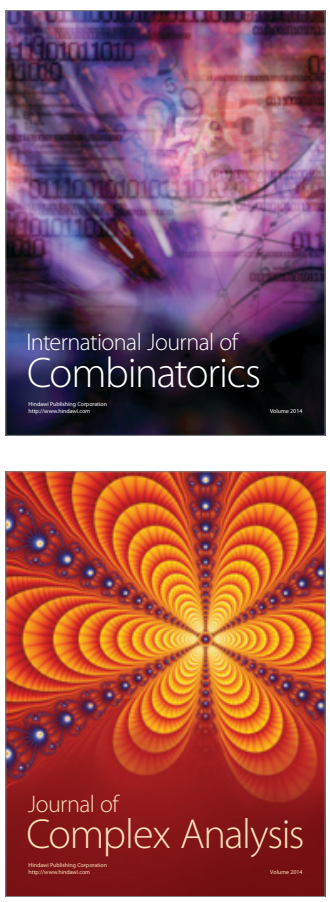

International Journal of

Mathematics and

Mathematical

Sciences
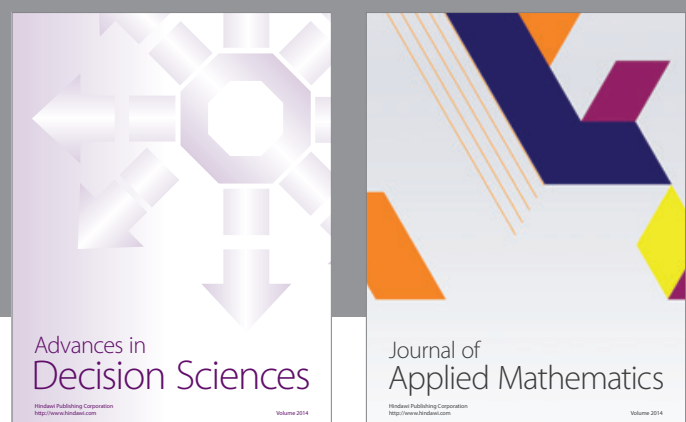

Journal of

Applied Mathematics
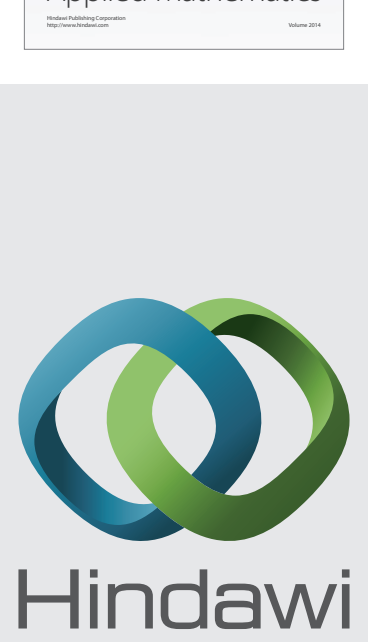

Submit your manuscripts at http://www.hindawi.com
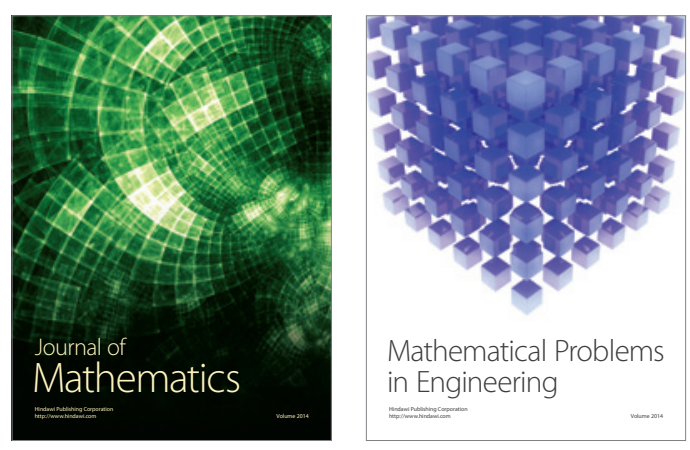

Mathematical Problems in Engineering
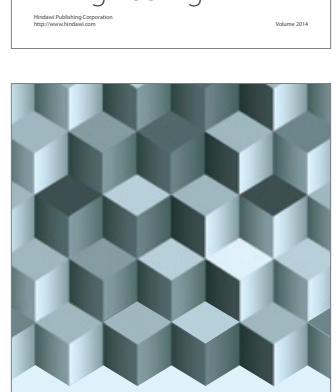

Journal of

Function Spaces
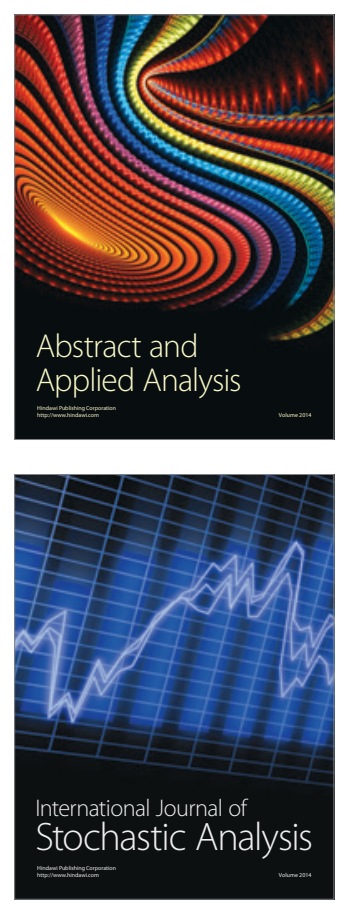

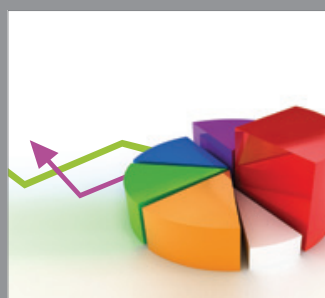

ournal of

Probability and Statistics

Promensencen
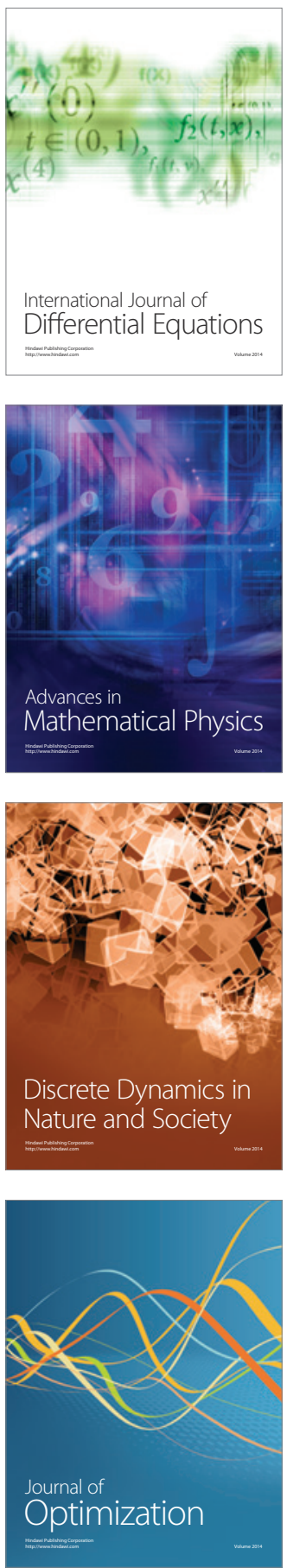